\title{
Distributed Adaptive Coordinated Control of Multiple Euler-Lagrange Systems considering Output Constraints and Time Delays
}

\author{
Hongde Qin, Xiaojia Li, and Yanchao Sun \\ Science and Technology on Underwater Vehicle Laboratory, Harbin Engineering University, Harbin 15001, China \\ Correspondence should be addressed to Yanchao Sun; sunyanchao@hrbeu.edu.cn
}

Received 4 February 2021; Accepted 3 May 2021; Published 19 May 2021

Academic Editor: Hamid Reza Karimi

Copyright (c) 2021 Hongde Qin et al. This is an open access article distributed under the Creative Commons Attribution License, which permits unrestricted use, distribution, and reproduction in any medium, provided the original work is properly cited.

\begin{abstract}
In this paper, we mainly investigate the coordinated tracking control issues of multiple Euler-Lagrange systems considering constant communication delays and output constraints. Firstly, we devise a distributed observer to ensure that every agent can get the information of the virtual leader. In order to handle uncertain problems, the neural network technique is adopted to estimate the unknown dynamics. Then, we utilize an asymmetric barrier Lyapunov function in the control design to guarantee the output errors satisfy the time-varying output constraints. Two distributed adaptive coordinated control schemes are proposed to guarantee that the followers can track the leader accurately. The first scheme makes the tracking errors between followers and leader be uniformly ultimately bounded, and the second scheme further improves the tracking accuracy. Finally, we utilize a group of manipulator networks simulation experiments to verify the validity of the proposed distributed control laws.
\end{abstract}

\section{Introduction}

With the rapid development of industrial technology, the industrial tasks are gradually becoming complicated and large-scale. When solving some complex industrial tasks, multiagent systems (MASs) gradually become the first choice due to its high reliability and economy, such as multiple robotic manipulator systems, spacecraft formation flying, and unmanned underwater vehicles [1-3]. More and more experts and scholars focus on the distributed coordinated control of MASs [4].

So far, there mainly have been two consensus methods for the MASs coordinated control. The first control strategy is about the leaderless control method, which requires all state variables to gradually converge to a constant. For example, the authors used Lyapunov finite-time theory to propose a consensus control method to ensure the states converge to a constant based on undirected graphs in [5]. The authors in [6] proposed a leaderless consensus control strategy and analysed the stability problem for MAS. However, for the leaderless case, the convergence of the state variables of all agents is related to the initial state variables of each agent, which leads to the great restriction of the system movement. The second strategy is the leader-following control for MASs. In this case, followers utilize their own or neighbors' information to track leader so that one only needs to design the movement of leader accurately. This method can not only simplify the design difficulty of the control algorithm but also reduce energy consumption and cost. Therefore, this approach is more suitable for MASs. In [7], a trajectory tracking algorithm was proposed by using the small gain feedback technique, so that the followers can effectively track the dynamic leader. In [8], the authors used the linear event-trigger feedback technique to make the state variables of followers and leaders tend to be consistent in finite time so as to achieve the tracking control. For the coordinated tracking control case, the leader's motion is the most crucial part in MASs, which means that the whole system will stop working if leader fails. Actually, virtual leaders play the same role as the real leaders in MASs. At the same time, virtual leaders can change the number of leaders flexibly [9]. In [10], the authors discussed the current 
situation and illustrated the extensive application prospect of the leadership relationship within virtual working environment. Furthermore, the input signals were regarded as the virtual leaders and a distributed control algorithm was proposed by using Lyapunov theory; this control algorithm realized the coordinated movement of the followers and achieved the goal of tracking the target trajectory $[11,12]$.

It is worth noting that all the above research studies about MASs are based on linear systems. However, linear systems have great limitations in MASs due to the existence of nonlinear uncertainties. Therefore, it is necessary to study the coordinated control problem of nonlinear systems $[13,14]$. In fact, Euler-Lagrange (EL) equation is widely used in the field of nonlinear systems, such as autonomous underwater robots and manipulating robots systems [15-17]. In [18], the authors focused on the distributed coordinated tracking issues and designed two kinds of novel control algorithms for multiple EL systems. In multiple EL systems, distributed observers are often used to ensure the normal operation of the systems when only partial agents could get the state information of the leader. In [19], the authors utilized a new dynamic velocity observer to ensure all agents could get the leader's velocity variables. In [20], the leader's information could be acquired by the followers through the velocity observer and guaranteed the goal of coordinated tracking control. At present, there usually exist communication delays among different agents due to the limitation of communication bandwidth and packet losses. Therefore, the communication delays cannot be ignored in the study of multiple EL systems' coordinated control [21]. Considering the communication delays, a new aperiodic sampled-data cluster formation control algorithm was proposed for the cluster formation control problem for MASs in [22]. In [23], the authors investigated the leader-following consensus problem of multiple EL systems which considered communication delays under the switching network. In [24], in order to solve the problem of communication delays, the authors proposed a novel coordinated tracking control algorithm based on Lyapunov stability theory. In actual engineering projects, state constraint problems or output constraint problems are often considered to meet certain requirements. At present, the state constraint or output constraint methods for nonlinear systems include the prescribed performance (PP) methods and the barrier Lyapunov function (BLF) technique [25]. PP methods mainly utilize error transformation to achieve the performance constraint $[26,27]$, which may cause control law singularity problem. In contrast, the BLF technique mainly applies the performance constraint variables to construct constrained boundary functions, which greatly reduces the difficulty of designing the control algorithms. Thus BLF technique is widely used in the state constraint control problem for nonlinear systems. In [28], the authors utilized Integral BLFs to make all state errors converge to the neighbor of origin. The authors used an asymmetric BLF to solve the timevarying output error limitation problem in [29]. The authors in [30] utilized the asymmetric time-varying BLF to construct an adaptive controller and applied the Lyapunov stability theory to guarantee the output error within the excepted range. It is feasible to use the time-varying BLF method to deal with the output constraints of multiple EL systems.

Generally speaking, MASs are often affected by working environment, unknown dynamics, and unknown disturbances. The uncertainties will affect the work efficiency of MASs. To handle the uncertainties problem, the neural network (NN) technique is widely used for the MASs due to its good approximation ability [31, 32]. In [33], the authors designed a distributed adaptive NN controller to ensure the mobile robots can obtain the expected control effect. In the case that only partial agents could get the leader's information, a control algorithm which used the $\mathrm{NN}$ technique to compensate the uncertainties and external disturbances was designed in [34]. It made the tracking errors among leader and followers tend to origin.

In this study, the coordinated control problems for nonlinear multiple EL systems considering communication delays and output constraints are investigated. Compared with the existing papers, it uses a distributed observer to solve the communication delay problem of multiple EL systems. Then, the BLF technique is used to guarantee the time-varying output errors of the systems within the prescribed constraint boundary. We also utilize adaptive NNs to deal with the uncertain dynamics and the unknown disturbances of the multiple EL systems. The main contributions of the paper are summarized as follows.

(1) Considering communication delays among different followers, the input signal source is regarded as a virtual leader. In addition, a distributed observer is used to ensure that the virtual leader's state information can be obtained by all followers.

(2) Two distributed control schemes are designed to guarantee the tracking errors are UUB and asymptotically converge to origin.

(3) The adaptive NN technique is utilized to compensate the uncertain dynamics of the multiple EL systems.

(4) Based on the BLFs, an asymmetric BLF is designed to make the output errors satisfy the time-varying output constraint requirements.

In the following research, Section 2 introduces the dynamic model and some basic lemmas. In Section 3, two distributed adaptive control algorithms and stability analysis are formulated. Section 4 presents several simulation examples to prove the validity of the control algorithms. Section 5 summarizes the whole paper.

\section{Materials and Methods}

2.1. The Basic Knowledge. The basic mathematical symbols and definitions in this paper are shown in Table 1.

The communication interactions among a virtual leader and $n$ followers can be presented by the directed graph $\zeta=(v, \varepsilon, A)$. In the directed graph $\zeta=(v, \varepsilon, A), u$ $\{1,2, \ldots, n+1\}$ is the set of nodes, $\varepsilon \subseteq v \times v$ denotes the set of edges, and $A=\left\{a_{i j}\right\} \in \mathbb{R}^{(n+1) \times(n+1)}$ represents the nonnegative adjacency matrix. In node set $v, v_{i}$ denotes the $i$ th 
TABLE 1: Mathematical symbols in this study.

\begin{tabular}{lc}
\hline Mathematical symbol & Definition \\
\hline $\mathbb{R}$ & The set of real numbers \\
$\mathbb{R}^{n}$ & The set of $n$-dimensional column vectors with all elements being real numbers \\
$\mathbb{R}^{n \times n}$ & The set of $n \times n$ real matrices \\
$I_{p}$ & $n \times n$ identity matrix \\
$\operatorname{diag}\left(x_{1}, \ldots, x_{n}\right)$ & Diagonal matrices whose diagonal elements are $x_{1}, \ldots, x_{n}$ \\
$\otimes$ & Kronecker product \\
$\lambda_{\min }(\cdot)$ & The minimum eigenvalue of a real symmetric matrix \\
$\lambda_{\max }(\cdot)$ & The maximum eigenvalue of a real symmetric matrix \\
$L_{p}$ & $\left\{f(t): \mathbb{R} \mapsto \mathbb{R}^{n} \mid\left(\int_{0}^{\infty}\|f(\tau)\|^{p}\right)^{1 / p}<\infty\right\}$ \\
$\operatorname{tr}(\cdot)$ & Matrix trace \\
\hline
\end{tabular}

follower. The edge $v, v_{i} \in \varepsilon$ represents that the $j$ th agent can get the information from the $i$ th agent. We call $v_{i}$ as the parent node and its neighbor $v_{j}$ is called as the child node. The path of a directed graph is the sequence of nodes $v_{i l}, \ldots, v_{i n}$, which satisfies $\left(v_{i k}, v_{i k+1}\right) \in \varepsilon$. The directed tree denotes a directed graph in which each node has only one parent node, but there is one root node which is different. When a directed tree contains all nodes of the graph, it can be described as a directed spanning tree. If the directed spanning tree is a part of the directed graph, it is said that the directed graph contains a directed spanning tree.

Assumption 1. A directed spanning tree is contained within $\zeta$.

We define the element $a_{i j}=1$, if and only if $\left(v_{j}, v_{i}\right) \in \varepsilon$ and otherwise $a_{i j}=0$. The element $a_{(n+1) j}=0$ when $j=1, \ldots, n+1$ A matrix is defined as $\bar{A}=\overline{a_{i j}} \in \mathbb{R}^{n \times n}$, where $\bar{a}_{i j}=a_{i j} / \sum_{k=1}^{n+1} a_{i k}, i, j \in\{1, \ldots, n\}$.

2.2. Dynamics of Euler-Lagrange Systems. The multiple EL systems contain $n$ followers and a virtual leader. Then, we utilize the EL equation to describe the dynamic model of the $i$ th follower as

$$
M_{i}\left(q_{i}\right) \ddot{q}_{i}+C_{i}\left(q_{i}, \dot{q}_{i}\right) \dot{q}_{i}+g_{i}\left(q_{i}\right)=\tau_{i}+\omega_{i},
$$

where $q_{i} \in \mathbb{R}^{p}, i=1, \ldots, n$ denotes the generalized coordinate, $\tau_{i} \in \mathbb{R}^{p}$ denotes the control input torque, $M_{i}\left(q_{i}\right) \in \mathbb{R}^{p \times p}$ represents the inertia matrix which is symmetric and positive definite, $C_{i}\left(q_{i} \dot{q}_{i}\right) \in \mathbb{R}^{p \times p}$ represent the centripetal and Coriolis torques, $g_{i}\left(q_{i}\right) \in \mathbb{R}^{p}$ denotes the gravitational force, and $\omega_{i} \in \mathbb{R}^{p}$ is the external disturbance. We assume $M_{i}\left(q_{i}\right), C_{i}\left(q_{i}, \dot{q}_{i}\right)$, and $g_{i}\left(q_{i}\right)$ are all unknown.

Assumption 2. The disturbance $\omega_{i}$ is bounded, which one satisfies $\left\|\omega_{i}\right\| \leq \gamma$, where $\gamma$ is a bounded positive constant.

The following properties of EL systems (1) are useful.

Property 1. The matrix $\left(M_{i}\left(q_{i}\right)-2 C_{i}\left(q_{i} \dot{q}_{i}\right)\right)$ is skew-symmetric, i.e., $\forall x_{i} \in \mathbb{R}^{p}, x_{i}^{T}\left(\dot{M}_{i}\left(q_{i}\right)-2 C_{i}\left(q_{i}, \dot{q}_{i}\right)\right) x_{i}=0$.

Property 2. $M_{i}\left(q_{i}\right)$ is bounded, and it satisfies $\underline{B} I_{p} \leq M_{i}\left(q_{i}\right) \leq \bar{B} I_{p}$, where $\bar{B}$ and $\underline{B}$ are positive constants.

We assume that $q_{n+1}$ can be represented as follows [35]:

$$
\begin{aligned}
\dot{v} & =S v, \\
q_{n+1} & =F v,
\end{aligned}
$$

where $v \in \mathbb{R}^{p}$ denotes the auxiliary state variable, $S \in \mathbb{R}^{p \times p}$ and $F \in R^{n \times p}$ are constant real matrices, and $q_{n+1}$ represents the state of the leader.

Assumption 3. When all followers can obtain $S$ and $F, v$ and $\dot{v}$ are bounded.

Remark 1. If the coefficient matrices $S, F$ and the auxiliary state variable $v$ are chosen appropriately, any desired trajectory can be formed by the leader.

\section{The Distributed Adaptive Trajectory Tracking Control Law Design}

3.1. Time-Varying BLF Design. The useful lemmas used in this study are shown as follows.

Lemma 1 (see [36]). If there is a continuous Lyapunov function $V_{(w, t)}$ which satisfies $\bar{w}_{1}(\|w\|) \leq V(|w, t|) \leq \bar{w}_{2}(\|w\|)$ and the derivative $\dot{V}(w, t)=d V(w, t) / d t$ satisfies $\dot{V}_{(w, t)} \leq-k V_{(w, t)}+c$, where $k$ and $c$ are positive constants, then the variable $w(t)$ is bounded.

Lemma 2 (see [37]). For $\forall B \in \mathbb{R}^{p \times p}$, if matrix $B$ is symmetric and positive definite, the following inequality can be obtained: $\forall y \in \mathbb{R}^{p}, \lambda_{\text {min }}\|y\|^{2} \leq y^{T} B y \leq \lambda_{\max }\|y\|^{2}$.

Lemma 3 (see [38]). $\psi(t)$ is a continuously differentiable function. For $\forall t \in[0, \infty)$, if $\psi(t) \psi(t) \leq \Phi$ hold, is bounded where $\Phi$ is a small positive constant, it means that $\psi(t)$ is bounded $\forall t \in[0, \infty)$.

Lemma 4 (see [39]). Consider $x \in \mathbb{R}$ and $|x|<\left|k_{a}\right|$, where $k_{a}$ is a positive constant, such that the inequality is shown as $\ln \left(k_{a}^{2} / k_{a}^{2}-x^{2}\right) \leq\left(x^{2} / k_{a}^{2}-x^{2}\right)$.

In this study, if the trajectory tracking errors are too large, it may cause undesired losses. Therefore, the output states of the followers should be limited. If the time-varying bounds are $\underline{k} c(t)=\left[\underline{k} c_{1}(t), \ldots, \underline{k} c_{n}(t)\right]^{T}$ and $\bar{k} c(t)=$ $\left[\bar{k} c_{1}(t), \ldots, \bar{k} c_{n}(t)\right]^{T}$, then the output $q_{i}(t)$ should remain in the region: 
$\Omega q_{i}:=\left\{q_{i}(t) \in R \mid \underline{k} c_{i}(t)<q_{i}(t)<\bar{k} c_{i}(t)\right\}, \quad \forall t \geq 0$.

When considering the influence of tracking errors on multiple EL systems, a time-varying BLF is used in this study to ensure that the output $q_{i}$ satisfies the time-varying output constraints.

First, an auxiliary variable is defined as

$$
q_{r i}=F \eta i .
$$

Then, we define the following error variables:

$$
\begin{aligned}
& Z_{1 i}=q_{i}-q_{r i}, \\
& Z_{2 i}=\dot{q}_{i}-r_{i},
\end{aligned}
$$

where $r_{i}$ denotes a virtual control.

Inspired by $\mathrm{He}$ et al. [40], we set the time-varying bounds of $Z_{1 i}$ as

$$
\begin{aligned}
k a_{i}(t) & =q_{r i}(t)-\underline{k} c_{i}(t), \\
k b_{i}(t) & =\bar{k} c_{i}(t)-q_{r i}(t) .
\end{aligned}
$$

Consider that asymmetric BLF is an improvement of symmetric BLF, which can better adapt to the requirements of time-varying output constraints and ensure the high trajectory tracking accuracy for multiple EL systems. An asymmetric BLF is chosen as

$$
V_{1 i}(t)=\frac{h(i)}{2} \operatorname{In} \frac{k b_{i}^{2}(t)}{k b_{i}^{2}(t)-\left\|Z_{1 i}(t)\right\|^{2}}+\frac{1-h(i)}{2} \operatorname{In} \frac{k a_{i}^{2}(t)}{k a_{i}^{2}(t)-\left\|Z_{1 i}(t)\right\|^{2}},
$$

where we define $h(i)$ as

$$
h(i)=\left\{\begin{array}{ll}
0, & Z_{1 i} \leq 0, \\
1, & Z_{1 i}>0,
\end{array} \quad i=1,2, \ldots, n .\right.
$$
follows:

The output tracking error variables are transformed as

$$
\begin{aligned}
\varepsilon_{a i} & =\frac{Z_{1 i}}{k a_{i}}, \\
\varepsilon_{b i} & =\frac{Z_{1 i}}{k b_{i}}, \\
\varepsilon_{i} & =h(i) \varepsilon_{b i}+(1-h(i)) \varepsilon_{a i} .
\end{aligned}
$$

Substituting (11) into (9), we obtain

$$
V_{1 i}(t)=\frac{1}{2} \operatorname{In} \frac{1}{1-\varepsilon_{i}^{2}} \text {. }
$$

From (12), we can know that $V_{1 i}(t)$ is positive definite and continuously differentiable when $\varepsilon_{i}<1$. Differentiating $V_{1 i}(t)$ yields

$$
\begin{aligned}
\dot{V}_{1 i}(t)= & \frac{\varepsilon_{b i} h(i)}{\left(1-\varepsilon_{b i}^{2}\right) k b_{i}}\left(Z_{2 i}+r_{i}-\dot{q}_{r i}-Z_{1 i} \frac{\dot{k} b_{i}}{k b_{i}}\right) \\
& +\frac{\varepsilon_{a i}(1-h(i))}{\left(1-\varepsilon_{a i}^{2}\right) k a_{i}}\left(Z_{2 i}+r_{i}-\dot{q}_{r i}-Z_{1 i} \frac{\dot{k} a_{i}}{k a_{i}}\right) .
\end{aligned}
$$

The virtual control $r_{i}$ is selected as

$$
r_{i}=\dot{q}_{r i}-k_{1 i} Z_{1 i}-\bar{K}_{1 i} Z_{1 i}
$$

where $\bar{K}_{1 i}$ is defined as

$$
\bar{K}_{1 i}=\sqrt{\beta+\left(\frac{\dot{k} a_{i}}{k a_{i}}\right)^{2}+\left(\frac{\dot{k} b_{i}}{k b_{i}}\right)^{2}},
$$

$\beta$ is a positive constant, and it can ensure $\dot{r}_{i}$ is bounded when $\dot{k} a_{i}$ and $\dot{k} b_{i}$ are zero, where the gain matrix $K_{1}=$ $\operatorname{diag}\left[k_{11}, k_{12}, \ldots, k_{1 i}, \ldots, k_{1 n}\right]$ is symmetric positive definite. Substituting (14) and (15) into (13) yields

$$
\begin{aligned}
\dot{V}_{1 i}(t)= & \frac{\varepsilon b_{i} h(i)}{\left(1-\varepsilon_{b_{i}}^{2}\right) k b_{i}}\left(Z_{2 i}+r_{i}-\dot{q}_{r i}-Z_{1 i} \frac{\dot{k} b_{i}}{k b_{i}}\right) \\
& +\frac{\varepsilon a_{i}(1-h(i))}{\left(1-\varepsilon_{a_{i}}^{2}\right) k a_{i}}\left(Z_{2 i}+r_{i}-\dot{q}_{r i}-Z_{1 i} \frac{\dot{k} a_{i}}{k a_{i}}\right) \\
\leq & -k_{1 i} \frac{\varepsilon_{i}^{2}}{1-\varepsilon_{i}^{2}}+X_{i} Z_{1 i} Z_{2 i},
\end{aligned}
$$

where $X_{i}$ is defined as follows:

$$
X_{i}=\left(\frac{h(i)}{k_{b_{i}}^{2}-\left\|Z_{1 i}\right\|^{2}}+\frac{1-h(i)}{k_{a_{i}}^{2}-\left\|Z_{1 i}\right\|^{2}}\right), \quad i=1,2,3, \ldots, n,
$$

and $X=\operatorname{diag}\left[X_{1}, X_{2}, \ldots, X_{i}, \ldots, X_{n}\right]$.

3.2. Distributed Adaptive NN Tracking Controller 1 Design. When only part of the followers can get the state information, we use the following distributed observer [35]: 


$$
\dot{\eta}_{i}=S\left(\eta_{i}-d_{i} \sum_{j=1}^{n+1} a_{i j} \eta_{j}(t-T)+d_{i} \sum_{j=1}^{n+1} a_{i j}\left(\dot{\eta}_{j}(t-T)+\eta_{j}\right)(t-T)-\eta_{i}\right),
$$

where $d_{i}=1 / \sum_{j=1}^{n+1} a_{i j}, \eta_{i r}=\mathbb{R}^{p}$ represents the $i$ th follower's estimate of $v$ and $T$ represents the constant time delays among different followers. We assume that $\eta_{n+1}=v$.

Lemma 5 (see [41]). In this research, we utilize the distributed observer (18) to ensure the leader's information can be acquired by all agents when Assumptions 1-3 hold. If $\|D \bar{\eta}\|>\lambda_{\max }(S)+\left\|R_{e}\right\|$ holds, we can draw a conclusion that $\eta_{i}-v$ is bounded, which can be expressed as

$$
\lim _{t \rightarrow \infty}\left\|\eta_{i}-v\right\| \leq U_{0}
$$

where $\quad R_{e}=-\left(I_{n} \otimes S\right) A\left[1_{n} \otimes v \quad(t-T)\right]-1_{n} \otimes v+A\left[1_{n} \otimes\right.$ $\dot{v}(t-T)]+A\left[1_{n} \otimes v(t-T)\right], \bar{\eta}=\left[\bar{\eta}_{1}^{T}, \ldots, \bar{\eta}_{n}^{T}\right]^{T}, \bar{\eta}_{i}=\eta_{i}-v$, $U_{0}$ denotes a positive constant, and $D \bar{\eta}=\bar{\eta}-A \bar{\eta}(t-T)$ is the defined neutral operator.

Remark 2. Since $S$ and $F$ are real matrices and the elements are independent of time and the state variables of the leader, we utilize matrices $S$ and $F$ to design the observer (18).

According to [42], we design following distributed adaptive control schemes of (1) as

$$
\begin{aligned}
\tau_{i} & =-K_{2 i} Z_{2 i}-X_{i} Z_{1 i}-\widehat{W}_{i}^{T} \phi_{i}, \\
\dot{\widehat{W}}_{i} & =\alpha\left(\phi_{i} Z_{2 i}^{T}-\mu \widehat{W}_{i}\right),
\end{aligned}
$$

where $\alpha$ and $\beta$ are two positive constants and $K_{2 i}$ is a gain matrix. $\widehat{W}_{i}$ and $\phi_{i}$ are two related variables to NN.

Theorem 1. For the EL system (1) which considers communication delays and output constrains, if Assumptions 1-3 hold, the tracking error $Z_{1 i}$ is UUB with the distributed observer (18) and the distributed adaptive control laws (20) and (21). At the same time, $q_{i}(t)$ satisfies the time-varying output constraints, i.e., $\forall t>0, \underline{k} c_{i}(t)<q_{i}(t)<\bar{k} c_{i}(t)$.

$$
\dot{Z}_{2 i}=\ddot{q}_{i}-\dot{r}_{i} .
$$

Substituting (22) into (1), we obtain

$$
M_{i} \dot{Z}_{2 i}+C_{i} Z_{2 i}=\tau_{i}+\omega_{i}+d_{i}\left(q_{i}, \dot{q}_{i}, r_{i}, \dot{r}_{i}\right),
$$

where

$$
d_{i}\left(q_{i}, \dot{q}_{i}, r_{i}, \dot{r}_{i}\right)=-g_{i}-C_{i} r_{i}-M_{i} \dot{r}_{i} .
$$

In this study, $M_{i}\left(q_{i}\right), C_{i}\left(q_{i}, \dot{q}_{i}\right), g_{i}\left(q_{i}\right)$ are assumed to be unknown, and there are nonlinear uncertainties in the EL system (1). Considering that $\mathrm{NN}$ has good approximation ability for unknown nonlinear function, it is often used to deal with the uncertainty problem in nonlinear system. Therefore, the NN technique is used to solve the nonlinear uncertainties $d_{i}\left(q_{i}, \dot{q}_{i}, r_{i}, \dot{r}_{i}\right)$. The methods are shown as follows:

$$
W_{i}^{T} \phi_{i}\left(q_{i}, \dot{q}_{i}, r_{i}, \dot{r}_{i}\right)=d_{i}\left(q_{i}, \dot{q}_{i}, r_{i}, \dot{r}_{i}\right)-\Delta_{i},
$$

where $W_{i}$ represents the ideal weighted matrix, $\phi_{i}$ is the Gauss function, and $\Delta_{i}$ denotes the approximation error. In this research, $\Delta_{i}$ is assumed to satisfy $\left\|\Delta_{i}\right\| \leq \Delta_{M i}$, where $\Delta_{M i}$ is a positive constant. The estimate of the nonlinear uncertainties $d_{i}$ for the $i$ th follower can be written as

$$
\widehat{d}_{i}\left(q_{i}, \dot{q}_{i}, r_{i}, \dot{r}_{i}\right)=\widehat{W}_{i}^{T} \phi_{i}\left(q_{i}, \dot{q}_{i}, r_{i}, \dot{r}_{i}\right),
$$

where $\widehat{W}_{i}$ is the estimate of $W_{i}$.

The distributed adaptive control schemes are designed as (20) and (21). Consider a Lyapunov function as follows:

$$
V_{2 i}=V_{1 i}+\frac{1}{2} Z_{2 i}^{T} M_{i} Z_{2 i}+\frac{1}{2 \alpha} \operatorname{tr}\left(\widetilde{W}_{i}^{T} \widetilde{W}_{i}\right),
$$

where $\widetilde{W}_{i}=W_{i}-\widetilde{W}_{i}$. Differentiating (27) and according to (20)-(26), we can obtain

Proof. Differentiating (6) yields

$$
\dot{V}_{2 i}=\dot{V}_{1 i}+Z_{2 i}^{T}\left(\omega_{i}+\Delta_{i}\right)+Z_{2 i}^{T} \tilde{W}_{i} \phi_{i}-\operatorname{tr}\left(\widetilde{W}^{T} \phi_{i} Z_{2 i}\right)+\mu \operatorname{tr}\left(\widetilde{W}_{i}^{T} \widehat{W}_{i}\right)
$$

Since $\Delta_{i}$ and $\omega_{i}$ are bounded, there is a positive constant $\bar{\chi}_{\max }$ satisfying $\left\|\Delta_{i}+\omega_{i}\right\| \leq \bar{\chi}_{\max }$. Furthermore, the inequality can be obtained as follows:

$$
Z_{2 i}^{T}\left(\omega_{i}+\Delta_{i}\right) \leq \frac{1}{2 \sigma} Z_{2 i}^{T} Z_{2 i}+\frac{\sigma}{2} \bar{\chi}_{\max }
$$

where $\sigma$ is a positive constant.

Because $Z_{2 i}^{T} \tilde{W}_{i} \phi_{i}$ is a scalar in (28), we have $Z_{2 i}^{T} \widetilde{W}_{i} \phi_{i}=\operatorname{tr}\left(Z_{2 i}^{T} \widetilde{W}_{i} \phi_{i}\right)=\operatorname{tr}\left(\widetilde{W}_{i}^{T} \phi_{i} Z_{2 i}\right)$.
The matrices have the following properties:

$$
\begin{aligned}
\operatorname{tr}(A+B) & =\operatorname{tr}(A)+\operatorname{tr}(B), \\
\operatorname{tr}(B A) & =\operatorname{tr}(A B), \\
2 \operatorname{tr}(A B) & \leq \operatorname{tr}\left(B^{T} B\right)+\operatorname{tr}\left(A^{T} A\right) .
\end{aligned}
$$

Further we have 


$$
\begin{aligned}
\operatorname{tr}\left(\tilde{W}_{i}^{T} \widehat{W}_{i}\right) & =\operatorname{tr}\left(\tilde{W}_{i}^{T} W_{i}\right)-\operatorname{tr}\left(\tilde{W}_{i}^{T} \tilde{W}_{i}\right) \\
& \leq \frac{1}{2} \operatorname{tr}\left(\tilde{W}_{i}^{T} \tilde{W}_{i}\right)+\frac{1}{2} \operatorname{tr}\left(W_{i}^{T} W_{i}\right)-\operatorname{tr}\left(\tilde{W}_{i}^{T} \tilde{W}_{i}\right) \\
& =\frac{1}{2} \operatorname{tr}\left(W_{i}^{T} W_{i}\right)-\frac{1}{2} \operatorname{tr}\left(\tilde{W}_{i}^{T} \tilde{W}_{i}\right) .
\end{aligned}
$$

$$
\dot{V}_{2 i} \leq \frac{\sigma}{2} \bar{\chi}_{\max }^{2}-\frac{1}{2}\left[\left(2 \lambda_{\min }\left(K_{2 i}\right)-\sigma^{-1}\right) Z_{2 i}^{T} Z_{2 i}+2 k_{1 i} \frac{\varepsilon_{i}^{2}}{1-\varepsilon_{i}^{2}}+\mu \operatorname{tr}\left(\tilde{W}_{i}^{T} \tilde{W}_{i}\right)-\mu \operatorname{tr}\left(W_{i}^{T} W_{i}\right)\right] .
$$

Thus, (34) can be written as

$$
\dot{V}_{2 i}(t) \leq-\kappa_{1} V_{2 i}+v_{1} \text {, }
$$

where

$$
\begin{aligned}
& \kappa_{1}=\min \left(\frac{2 \lambda_{\min }\left(K_{2 i}\right)-\sigma^{-1}}{\bar{B}}, \alpha, \mu, \min \left(2 k_{1 i}\right)\right), \\
& v_{1}=\frac{\mu}{2} \operatorname{tr}\left(W_{i}^{T} W_{i}\right)+\frac{\sigma}{2} \bar{\chi}_{\max }^{2} .
\end{aligned}
$$

We can know $V_{2 i}$ is UUB according to Lemma 1. Integrating (35) yields

$$
V_{2 i}(t) \leq V_{2 i}(0) e^{-\kappa_{1} t}+\frac{v_{1}}{\kappa_{1}}\left(1-e^{-\kappa_{1} t}\right) .
$$

We can ensure $\kappa_{1}>0$ and $v_{1}>0$ by properly selecting parameters, and we have

$$
\lim _{t \longrightarrow \infty} V_{2 i}(t)=\frac{v_{1}}{\kappa_{1}}=\rho .
$$

It can be seen from (27) that

$$
V_{1 i} \leq V_{2 i}
$$

Moreover,

$$
\lim _{t \rightarrow \infty} \frac{1}{2} \ln \frac{1}{1-\varepsilon_{i}^{2}} \leq \rho .
$$

By substituting (10) and (11) into (40), we can obtain $-\sqrt{k a_{i}^{2}(t)\left(1-e^{-2 p}\right)} \leq \lim _{t \rightarrow \infty}\left\|Z_{1 i}\right\| \leq \sqrt{k b_{i}^{2}(t)\left(1-e^{-2 p}\right)}$.

From (4), one has

$q_{i}-q_{n+1}=q_{i}-F \eta_{i}+F \eta_{i}-q_{n+1}=q_{i}-F \eta_{i}+F\left(\eta_{i}-v\right)$.

According to (19), (41), and (42), we can obtain

$$
\begin{aligned}
\lim _{t \longrightarrow \infty}\left\|q_{i}-q_{n+1}\right\| & \leq \lim _{t \longrightarrow \infty}\left\|q_{i}-F \eta_{i}\right\|+\|F\| \lim _{t \longrightarrow \infty}\left\|\eta_{i}-v\right\| \\
& \leq \sqrt{k b_{i}^{2}(t)\left(1-e^{-2 \rho}\right)}+U_{0}\|F\| .
\end{aligned}
$$

From (46), we can conclude that the tracking errors among different followers and the virtual leader are bounded.

From (5), (7), (8), and (41), we can obtain

$$
\underline{k} c_{i}(t)<q_{i}(t)<\bar{k} c_{i}(t)
$$

Equation (44) shows that the output $q_{i}(t)$ satisfies the time-varying output constraints.

\subsection{Improved Distributed Adaptive NN Tracking Controller 2} Design. According to [42], the authors proposed a new algorithm to decrease the tracking errors in (47) by using a discontinuous sign function, and it can be proved by similar steps to (49)-(58). However, this approach will bring about the additional chattering. Considering the chattering problem, we propose an improved continuous control scheme to improve the tracking accuracy. At the same time, auxiliary variable $Z_{i}(t)$ can converge to origin asymptotically.

For the EL system (1), we propose following modified distributed adaptive control strategy based on controller 1 as

$$
\begin{aligned}
\tau_{i} & =-K_{2 i} Z_{2 i}-X_{i} Z_{1 i}-\widehat{W}_{i}^{T} \phi_{i}-\iota_{i} \operatorname{sat}\left(Z_{2 i}\right), \\
\dot{W}_{i} & =\alpha \phi_{i} Z_{2 i}^{T},
\end{aligned}
$$

where $t_{i}$ is a positive constant, and it satisfies that

$$
\iota_{i} \geq\left\|\omega_{i}+\Delta_{i}\right\|
$$

$$
\operatorname{sat}\left(Z_{2 i}\right)= \begin{cases}\operatorname{sgn}\left(\frac{Z_{2 i}}{\delta}\right), & \left\|\frac{Z_{2 i}}{\delta}\right\| \geq 1, \\ \frac{Z_{2 i}}{\delta}, & \text { otherwise, }\end{cases}
$$

where $\delta$ is a small positive constant.

Remark 3. By introducing the continuous function sat $\left(Z_{2 i}\right)$, it not only makes the tracking errors among different followers and the leader asymptotically converge to zero but 
also avoids the chattering phenomenon caused by discontinuous sign function $\operatorname{sgn}\left(Z_{2 i} / \delta\right)$.

Theorem 2. For the EL system (1) which considers communication delays and output constrains, when Assumptions 1-3 hold, each follower can gradually track the leader and the tracking error $Z_{1 i}$ asymptotically converging to origin with the distributed continuous control laws (45) and (46). The timevarying output constraints are not breached, that is, $\forall t>0, \underline{k} c_{i}(t)<q_{i}(t)<\bar{k} c_{i}(t)$.

Proof. When $\left\|Z_{2 i} / \delta\right\|>1$, we know sat $\left(Z_{2 i}\right)=\operatorname{sgn}\left(Z_{2 i} / \delta\right)$. Substituting (45) and (46) into (1), we can obtain

$$
M_{i} \dot{Z}_{2 i}+C_{i} Z_{2 i}=-X_{i} Z_{1 i}-K_{2 i} Z_{2 i}+\bar{W}_{i}^{T} \phi_{i}+\omega_{i}+\Delta_{i}-\iota_{i} \operatorname{sgn}\left(\frac{Z_{2 i}}{\delta}\right) .
$$

Select the same $V_{1 i}$ and $V_{2 i}$ as those in Theorem 1. Taking the derivative of $V_{2 i}$ and from (16), (47), and (48), it can be obtained that

$$
\begin{aligned}
\dot{V}_{2 i} \leq & -Z_{2 i}^{T} K_{2 i} Z_{2 i}-k_{1 i} \frac{\varepsilon_{i}^{2}}{1-\varepsilon_{i}^{2}}+Z_{2 i}^{T}\left(\omega_{i}+\Delta_{i}\right) \\
& +Z_{2 i}^{T} \widetilde{W}_{i} \phi-\operatorname{tr}\left(\widetilde{W}_{i}^{T} \phi Z_{2 i}\right)-\iota_{i}\left\|\frac{Z_{2 i} \|}{\delta}\right\| .
\end{aligned}
$$

Similar to (28)-(34), it holds that

$$
\dot{V}_{2 i} \leq-Z_{2 i}^{T} K_{2 i} Z_{2 i}-k_{1 i} \frac{\varepsilon_{i}}{1-\varepsilon_{i}^{2}}+\left\|Z_{2 i}\right\|\left\|\omega_{i}+\Delta_{i}\right\|-\iota_{i}\left\|\frac{Z_{2 i}}{\delta}\right\| \text {. }
$$

For a small positive constant $\delta$, we can find that $\left\|Z_{2 i}\right\| \leq\left\|Z_{2 i} / \delta\right\|$. Substituting (47) into (51), we have

$$
\dot{V}_{2 i} \leq-Z_{2 i}^{T} K_{2 i} Z_{2 i}-k_{1 i} \frac{\varepsilon_{i}^{2}}{1-\varepsilon_{i}^{2}} .
$$

Since $k_{1 i}$ is a positive constant and matrix $K_{2 i}$ is symmetric positive definite, it can be found that

$$
\dot{V}_{2 i} \leq 0 \text {. }
$$

Moreover, we have

$$
\lim _{t \rightarrow \infty} V_{2 i}(t)=V_{\infty} \in[0, V(0)] .
$$

Due to the fact that $V_{2 i}$ is bounded, we find $Z_{\mu}, Z_{2 i} \in L_{\infty}$. According to (6), we have $\dot{Z}_{1 i} \in L_{\infty}$. When $\left|\varepsilon_{i}\right|<1$, $\left(\varepsilon_{i}^{2} /\left(1-\varepsilon_{i}^{2}\right)\right) \geq \varepsilon_{i}^{2}$. Integrating both sides of (54), it can be obtained that

$$
k_{1 i} \int_{0}^{\infty} \frac{\varepsilon_{i}^{2}}{1-\varepsilon_{i}^{2}} \mathrm{~d} t+\lambda_{\min }\left(K_{2 i}\right) \int_{0}^{\infty} Z_{2 i}^{T} Z_{2 i} \mathrm{~d} t \leq V_{2 i}(0)-V_{2 i}(\infty)
$$

Therefore, we have $Z_{1 i} \in L_{2}$ and $Z_{1 i} \in L_{2} \cap L_{\infty}$. According to Barbalet lemma, we have

$$
\lim _{t \longrightarrow \infty} Z_{1 i}(t)=0 \text {. }
$$

From (56), it can be found that

$$
\lim _{t \longrightarrow \infty}\left\|q_{i}-F \eta_{i}\right\|=0
$$

Following the similar steps as (42) and (43), we have

$$
\lim _{t \longrightarrow \infty}\left\|q_{i}-q_{n+1}\right\| \leq \lim _{t \longrightarrow \infty}\left\|q_{i}-F \eta_{i}\right\|+\|F\| \lim _{t \longrightarrow \infty}\|\eta-v\| \leq U_{0}\|F\| .
$$

The $i$ th follower has a good tracking performance on the virtual leader, and the upper boundary of the tracking error is described by (58).

When $\left\|Z_{2 i} / \delta\right\|<1$, it can be obtained that $\operatorname{sat}\left(Z_{2 i}\right)=Z_{2 i} / \delta$. According to (49) and (50), we have

$$
\begin{aligned}
\dot{V}_{2 i}(t) & \leq-Z_{2 i}^{T} K_{2 i} Z_{2 i}-k_{1 i} \frac{\varepsilon_{i}^{2}}{1-\varepsilon_{i}^{2}}-Z_{2 i}^{T}\left[\iota_{i} \frac{Z_{2 i}}{\delta}-\left(\omega_{i}+\Delta_{i}\right)\right] \\
& \leq-Z_{2 i}^{T} K_{2 i} Z_{2 i}-k_{1 i} \frac{\varepsilon_{i}^{2}}{1-\varepsilon_{i}^{2}}-\|Z\|\left[\frac{Z_{2 i}}{\delta}-\left\|\omega_{i}+\Delta_{i}\right\|\right] .
\end{aligned}
$$

If $\delta \leq \iota_{i}\left\|Z_{2 i}\right\| /\left\|\omega_{i}+\Delta_{i}\right\|$, we have $\dot{V}_{2 i}(t) \leq-Z_{2 i}^{T}$ $K_{2 i} Z_{2 i}-k_{1 i}\left(\varepsilon_{i}^{2} /\left(1-\varepsilon_{i}^{2}\right)\right)$. From the procedures in (53)-(58), we can find that the tracking error between the $i$ th follower and the virtual leader is bounded.

According to (5), (7), (8), and (56), we can obtain the same result as (44). It shows that the output $q_{i}(t)$ satisfies the time-varying output constraints.

Remark 4. The results of this paper can provide some reference for formation control of MASs. In the future, the formation control problems for MASs considering timevarying communication delays and full-state constraints will be studied.

\section{Results and Discussion}

4.1. Parameter Setting. In this section, we utilize some examples to verify the validity of the proposed schemes in practical aspect. In this study, we consider 4 2-degree-offreedom robotic manipulators as an example for the simulation experiment. The communication topology is shown in Figure 1, where 5 represents the virtual leader and 1-4 represent the four followers. The structure of robotic manipulator is shown in Figure 2.

The dynamic equation of the $i$ th follower can be expressed as

$$
M_{i}\left(q_{i}\right) \ddot{q}_{i}+C_{i}\left(q_{i}, \dot{q}_{i}\right) \dot{q}_{i}+g_{i}\left(q_{i}\right)=\tau_{i}+w_{i}, \quad i=1, \ldots, 4,
$$

where 


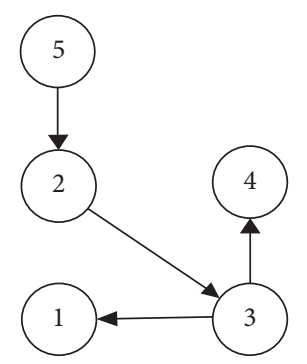

Figure 1: Communication topology.

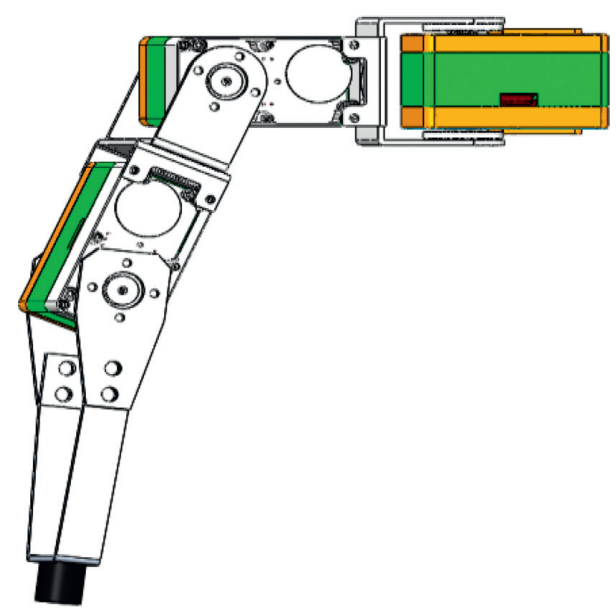

Figure 2: The structure of robotic manipulator.

$$
\begin{aligned}
q_{i} & =\left[q_{i 1}, q_{i 2}\right]^{T}, \\
M_{i}\left(q_{i}\right) & =\left[\begin{array}{cc}
\Xi_{i 1}+\Xi_{i 2}+2 \Xi_{i 3} \cos q_{i 2} & \Xi_{i 2}+\Xi_{i 3} \cos q_{i 2} \\
\Xi_{i 2}+\Xi_{i 3} \cos q_{i 2} & \Xi_{i 2}
\end{array}\right], \\
C_{i}\left(q_{i}, \dot{q}_{i}\right) & =\left[\begin{array}{cc}
-\Xi_{i 3}\left(\sin q_{i 2}\right) \dot{q}_{i 2} & -\Xi_{i 3}\left(\sin q_{i 2}\right)\left(\dot{q}_{i 1}+\dot{q}_{i 2}\right) \\
\Xi_{i 3}\left(\sin q_{i 2}\right) \dot{q}_{i 1} & 0
\end{array}\right], \\
g_{i}\left(q_{i}\right) & =\left[\begin{array}{c}
\Xi_{i 4} \cos \dot{q}_{i 1}+\Xi_{i 5} g \cos \left(q_{i 1}+q_{i 2}\right) \\
\Xi_{i 5} g \cos \left(q_{i 1}+q_{i 2}\right)
\end{array}\right], \\
\omega_{i} & =5 \% \cdot\left[M_{i}\left(q_{i}\right) \ddot{q}_{i}+C_{i}\left(q_{i}, \dot{q}_{i}\right) \dot{q}_{i}+g_{i}\left(q_{i}\right)\right] .
\end{aligned}
$$

In this section, $q_{1 i}, q_{2 i}$ represent the rotation angle of two joints of the manipulator. $\Xi_{i 1}=J_{i 1}+m_{i 2} 2_{i 1}^{2}, \quad \Xi_{i 1}=$ $0.25 m_{i 2} l_{i 2}^{2}+J_{i 2}, \quad \Xi_{i 3}=0.5 m_{i 2} l_{i 1} l_{i 2}, \quad \Xi_{i 4}=\left(0.5 m_{i 1}+m_{i 2}\right) l_{i 1}$, $\Xi_{i 5}=0.5 m_{i 2} l_{i 2}^{2}$, and $g=9.8 \mathrm{~m} / \mathrm{s}^{2}$ denotes gravitational acceleration, where $m_{i 1}$ and $m_{i 2}$ denote masses of links, $l_{i 1}$ is the length of links, $l_{i 2}$ represents the distance from center of mass to motors, and $J_{i 1}$ and $J_{i 2}$ are moments of inertia.

Table 2 shows the indicators of robotic manipulators.

We set the time-varying output constraints as follows:

$$
\begin{aligned}
& \bar{k}_{c}(t)=\left[2.2+15 e^{-0.8 t}, 2.2+15 e^{-0.5 t}, 2.2+15 e^{-0.35 t}, 2.2+15 e^{-0.25 t}\right]^{T}, \\
& \underline{k}_{c}(t)=\left[1.8+15 e^{-0.8 t}, 1.8+15 e^{-0.5 t}, 1.8+15 e^{-0.35 t}, 1.8+15 e^{-0.25 t}\right]^{T} . \\
& -k_{a i}(t)<Z_{1 i}(t)<k_{b i}(t) .
\end{aligned}
$$

The boundary value of tracking error $Z_{1 i}$ is expressed as

$$
\begin{aligned}
& k_{a i}(t)=q_{r i}(t)-\underline{k}_{c i}(t), \\
& k_{b i}(t)=\bar{k}_{c i}(t)-q_{r i}(t) .
\end{aligned}
$$

Thus, we have
The initial angle of robotic manipulators is set as follows. $q_{11}(0)=\pi / 5, \quad q_{12}(0)=-\pi / 3, \quad q_{21}(0)=2 \pi / 5, \quad q_{22}(0)=$ $-\pi / 6, q_{31}(0)=3 \pi / 5, q_{32}(0)=\pi / 6, q_{41}(0)=4 \pi / 5, q_{42}(0)=$ $\pi / 3$, and $\dot{q}_{i 1}(0)=\dot{q}_{i 2}(0)=0$.

For the $i$ th follower $(i=1, \ldots, 4)$, the activation 
TABLE 2: The parameters of the robotic manipulators.

\begin{tabular}{lcccc}
\hline Parameter & Manipulator 1 & Manipulator 2 & Manipulator 3 & Manipulator 4 \\
\hline$m_{i 1}(\mathrm{~kg})$ & 1.01 & 0.96 & 1.03 & 1.04 \\
$m_{i 2}(\mathrm{~kg})$ & 1.12 & 1.14 & 0.98 & 1.09 \\
$l_{i 1}(\mathrm{~m})$ & 1 & 0.95 & 0.95 & 1 \\
$l_{i 2}(\mathrm{~m})$ & 0.96 & 1 & 0.19 & 1 \\
$J_{i 1}\left(\mathrm{~kg} \cdot \mathrm{m}^{2}\right)$ & 0.23 & 0.21 & 0.42 & 0.21 \\
$J_{i 2}\left(\mathrm{~kg} \cdot \mathrm{m}^{2}\right)$ & 0.41 & 0.40 & & 0.41 \\
\hline
\end{tabular}

equation of the neural network system can be written as

$$
\phi_{i}(Z)=\left[\phi_{i 1}(z), \ldots, \phi_{i 6}(z)\right]^{T} .
$$

We select Gauss function as the activation function, and its form is

$$
\phi_{i j}(z)=\exp \left(\frac{\left\|z-c_{i j}\right\|^{2}}{\sigma_{i j}^{2}}\right), \quad j=1, \ldots, 6,
$$

where $z=\left[q_{i}, \dot{q}_{i}, r_{i}, \dot{r}_{i}\right]^{T} \in \mathbb{R}^{8}$. Suppose all followers utilize the same activation equation. $C_{i j}$ denotes the NN center and it is distributed over $[-5,5]^{4} \times[-0.5,0.5]^{4} . \sigma_{i j}$ is Gaussian function's width, and we define $\sigma_{i j}=2, \widehat{W}_{i}(0)=0_{6 \times 2}$.

The desired trajectory of the virtual leader is designed as follows:

$$
\begin{aligned}
& q_{51}(t)=q_{51 \_\mathrm{amp}} \sin \left(\omega t+\phi_{51 \_\mathrm{pha}}\right)+q_{51 \_ \text {bias }}, \\
& q_{52}(t)=q_{52 \_\mathrm{amp}} \sin \left(\omega t+\phi_{52_{\text {_pha }}}\right)+q_{52 \text { _bias }},
\end{aligned}
$$

where $\quad q_{51 \_ \text {amp }}=\pi / 6, \quad q_{51 \text { _pha }}=\pi / 2, \quad q_{51 \_ \text {bias }}=\pi / 2$, $q_{52_{\text {_amp }}}=2 \pi / 3, \phi_{52_{\text {_pha }}}=\pi / 2, q_{52_{\text {_bias }}}=0$, and $\omega=0.1 \pi$.

The state variable $q_{5}$ of the virtual leader can be expressed as

$$
\begin{gathered}
\dot{v}=S v, \\
q_{5}=F v,
\end{gathered}
$$

where

$$
\begin{aligned}
& v=\left[\begin{array}{c}
\sin (\omega t) \\
\sin \left(\omega t+\frac{\pi}{2}\right) \\
1
\end{array}\right], \\
& \omega=0.1 \pi, \\
& S=\left(\begin{array}{ccc}
0 & 0.1 \pi & 0 \\
-0.1 \pi & 0 & 0 \\
0 & 0 & 0
\end{array}\right) \text {, } \\
& F=\left(\begin{array}{ccc}
0 & \frac{\pi}{6} & \frac{\pi}{2} \\
& \frac{2 \pi}{3} & 0
\end{array}\right) .
\end{aligned}
$$

In practical physical systems, we often need to limit the amplitude of the control inputs $\tau_{i}$. The following saturation equation is used to limit the amplitude:

$$
\operatorname{sat}\left(\tau_{i}\right)=\left\{\begin{array}{l}
-\tau_{i \max } \tau_{i}<-\tau_{i \max }, \\
\tau_{i}\left|\tau_{i}\right| \leq \tau_{i \max }, \\
\tau_{i \max } \tau_{i}>\tau_{i \max },
\end{array}\right.
$$

where $\tau_{i \max }$ denotes a positive constant, and we let $\tau_{i \max }=50 \mathrm{Nm}$.

In this section, we choose the communication delay as $T=0.5 \mathrm{~s}$

4.2. Simulation Performance for Controller 1. For controller 1 , we choose the parameters as $k_{1 i}=10, K_{2 i}=20 I_{2}, \gamma=1$, and $v=10$. Figures 3-12 introduce the simulation results.

Figures 3 and 4 illustrate the state variables $q_{i 1}$ and $q_{i 2}$ among the followers and the virtual leader, from which we can see that each follower can effectively track the leader after about $5 \mathrm{~s}$. Figures 5 and 6 show that the auxiliary variables $Z_{i 1}$ and $Z_{i 2}$ are bounded. At the same time, the auxiliary variables $Z_{i 1}$ do not exceed $0.1 \mathrm{rad}$, and $Z_{2 i}$ do not exceed 0.05 after 3 s. From Figures 7 and 8, it can be obtained that the control inputs are continuous and change within $10 \mathrm{Nm}$. From Figures 9-12, we can find that the time-varying output constraints of the robotic manipulators are always satisfied.

4.3. Simulation Performance for Algorithm 2. For the improved algorithm by using discontinuous sign function on Algorithm 1, let $k_{1 i}=20, K_{2 i}=20 I_{2}, \gamma=1, \iota_{i}=80$, and $\delta=0.5$. Figures 13 and 14 show that the control input torque of all robotic manipulators has chattering problem.

For Algorithm 2, we set $k_{1 i}=20, K_{2 i}=20 I_{2}, \gamma=1$, $\iota_{i}=80$, and $\delta=0.5$. Figures $15-24$ show the performance of simulation results.

From Figures 15 and 16, it is obvious that the tracking performance is better, and the tracking errors are smaller compared with the cases in Figures 5 and 6. Comparing Figures 17 and 18 with Figures 7 and 8, we can find that the fluctuation range of $Z_{1 i}$ and $Z_{2 i}$ is smaller after $1 \mathrm{~s}$. From Figures 19 and 20, we can obtain that the control input torques of the robotic manipulators are stable and continuous. From Figures 21-24, we can see that under the control Algorithm 2, the error variables of the followers always satisfy the time-varying output constraints.

Based on the above simulation results, we can discover that all followers have very good tracking effect on the leader, 


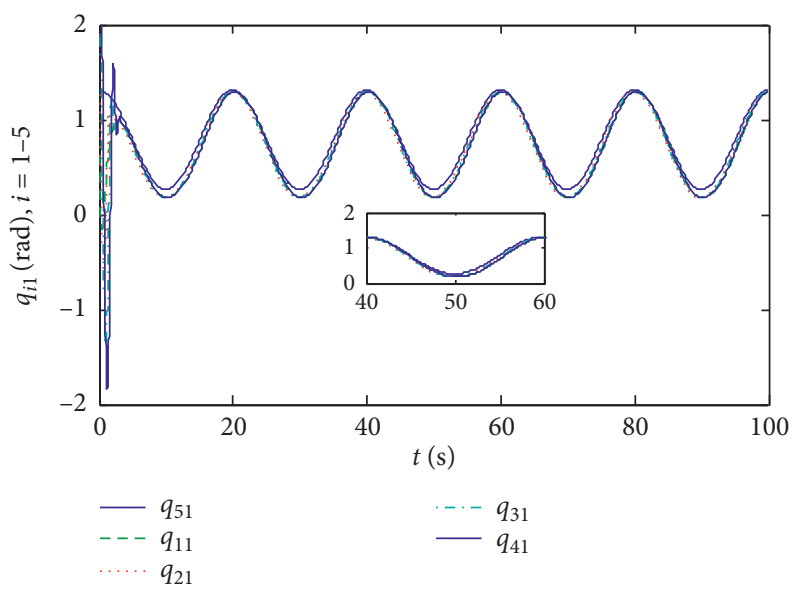

FIgURE 3: The generalized coordinates $q_{i 1}, i=1, \ldots, 4$ of manipulators.

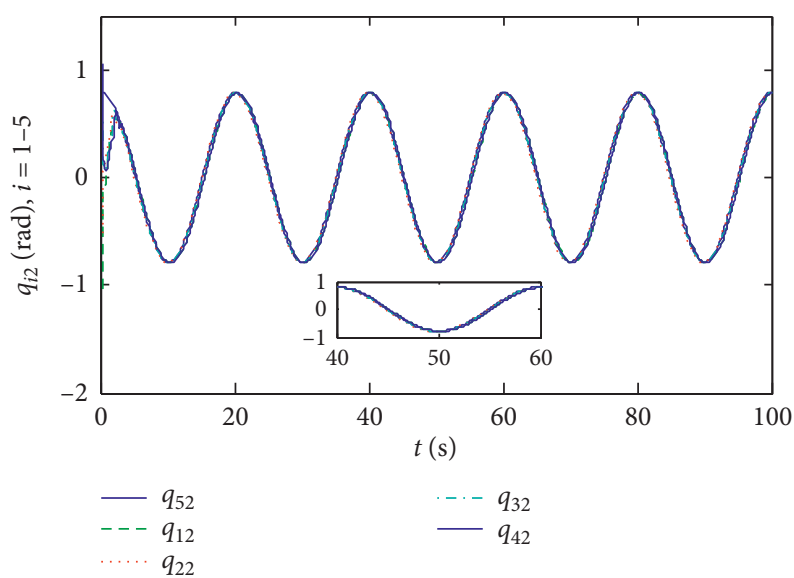

FIGURE 4: The generalized coordinates $q_{i 2}, i=1, \ldots, 4$ of manipulators.

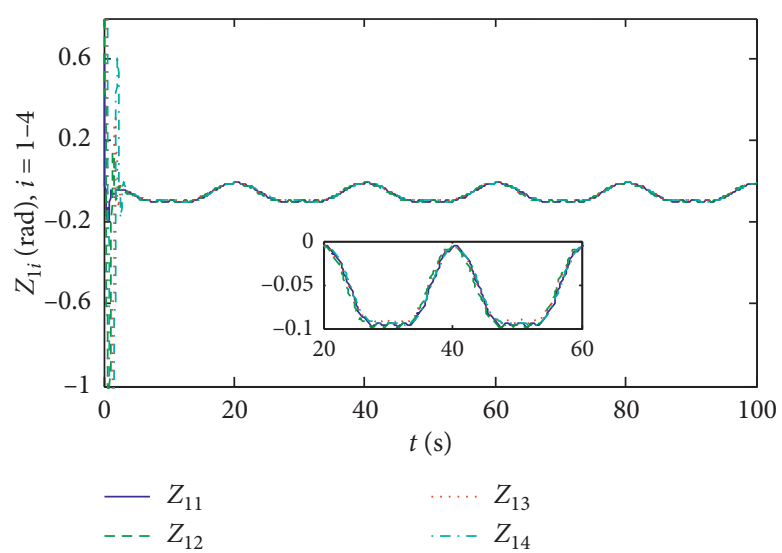

Figure 5: The auxiliary variables $Z_{i 1}, i=1, \ldots, 4$. 


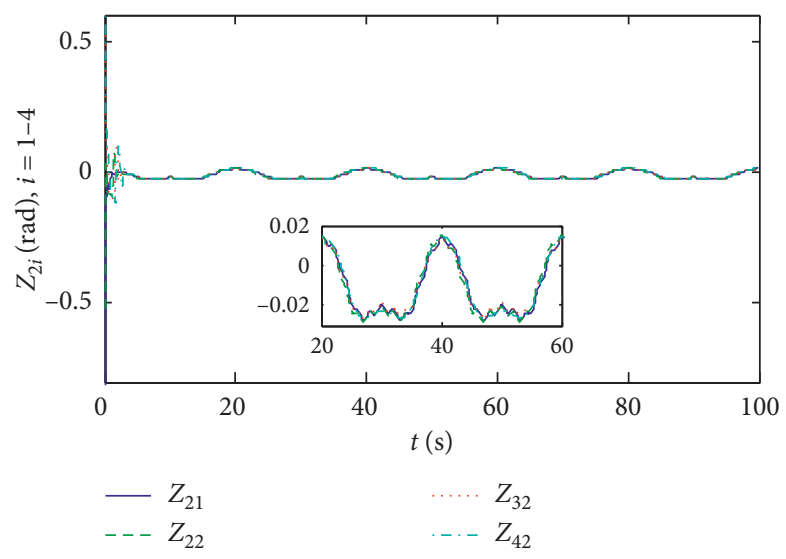

FIgURE 6: The auxiliary variables $Z_{i 2}, i=1, \ldots, 4$.

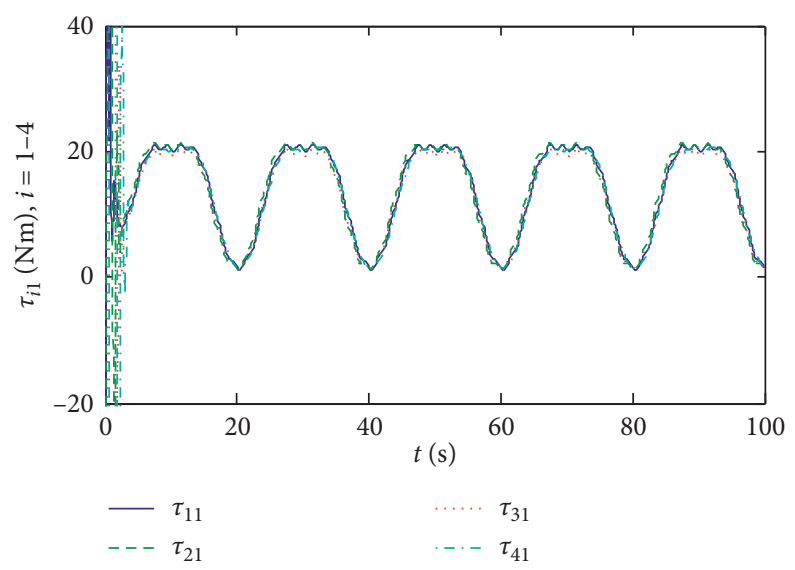

FIgURE 7: The control input torque $\tau_{i 1}, i=1, \ldots, 4$ of manipulators.

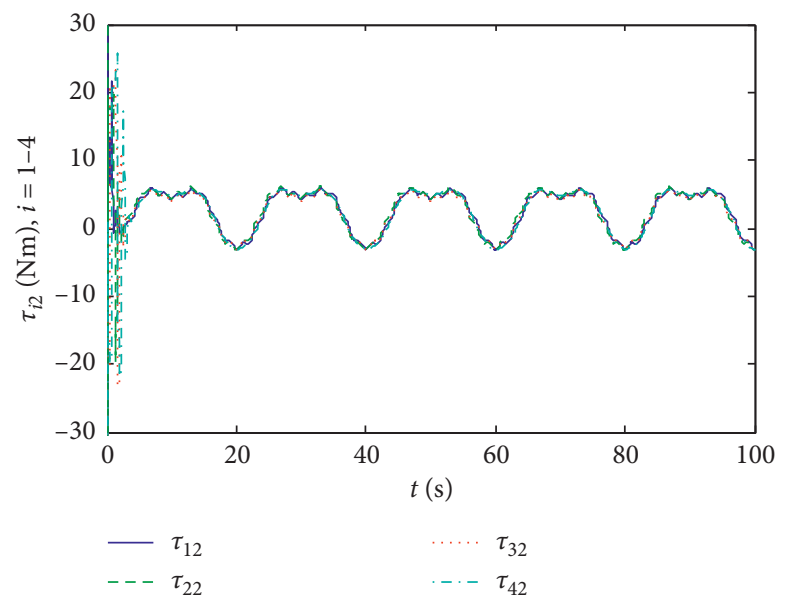

FIgURE 8: The control input torque $\tau_{i 2}, i=1, \ldots, 4$ of manipulators. 


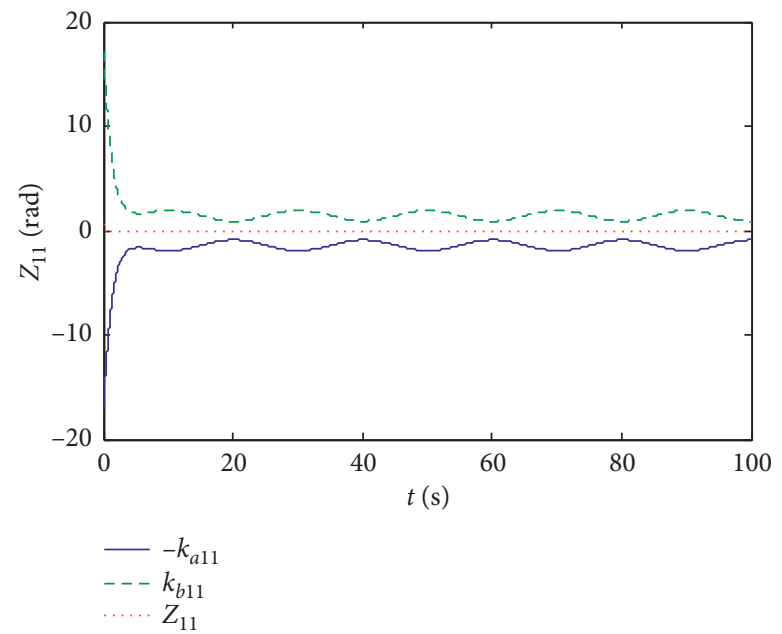

Figure 9: Constraint effect of $Z_{11}$ under Algorithm 1.

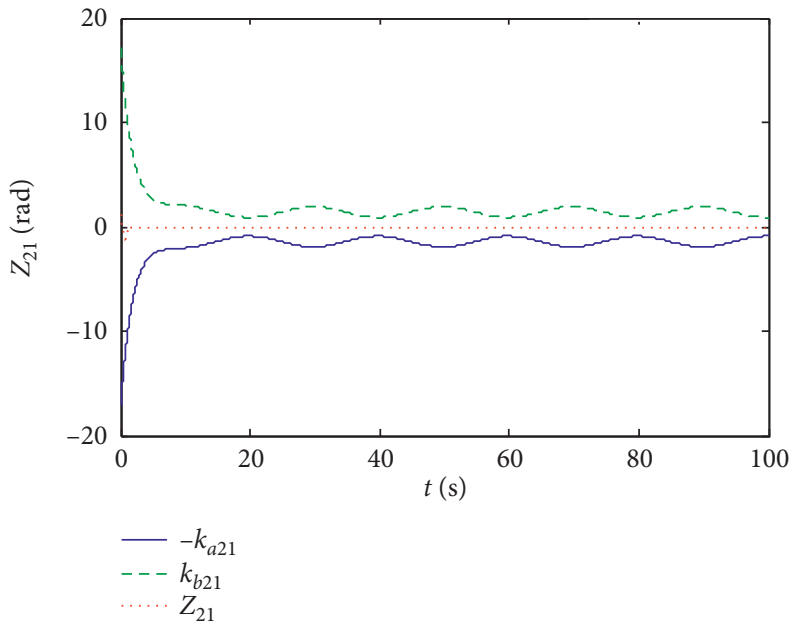

Figure 10: Constraint effect of $Z_{21}$ under Algorithm 1.

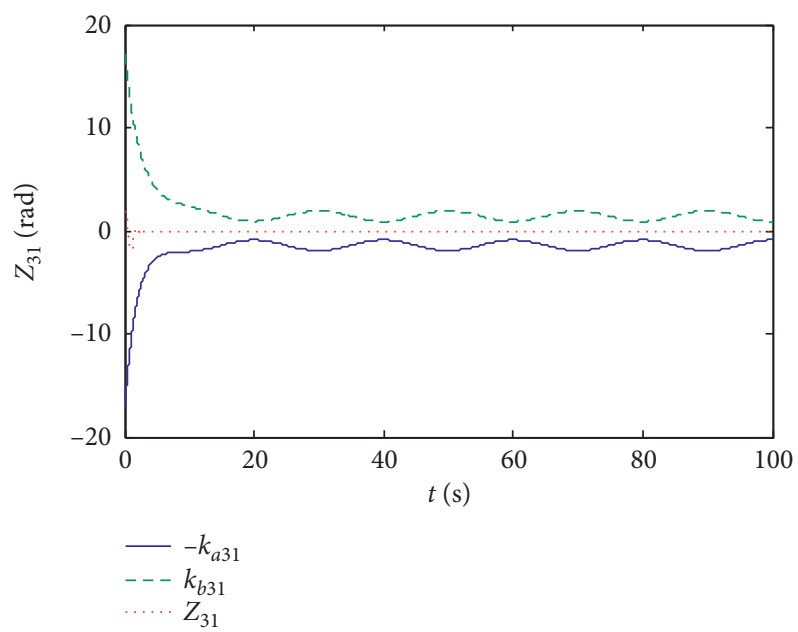

Figure 11: Constraint effect of $Z_{31}$ under Algorithm 1. 


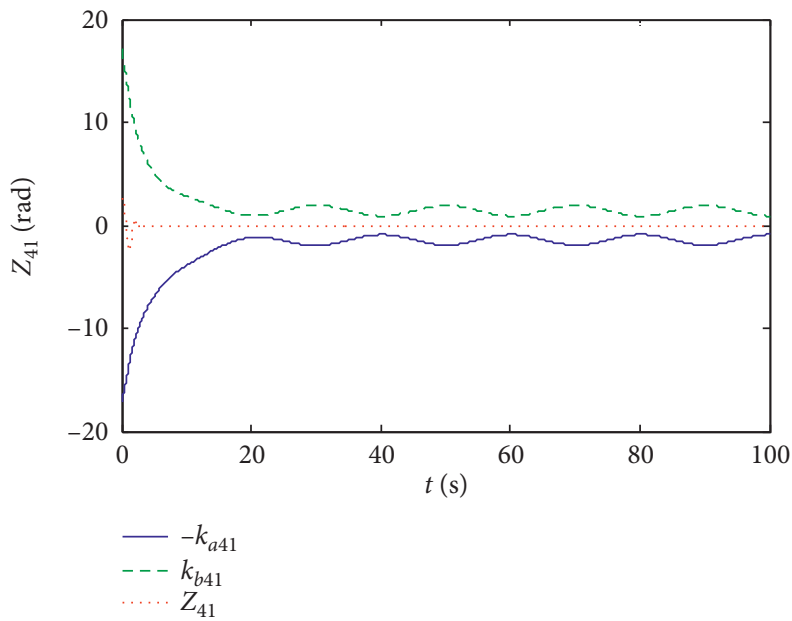

Figure 12: Constraint effect of $Z_{41}$ under Algorithm 1.

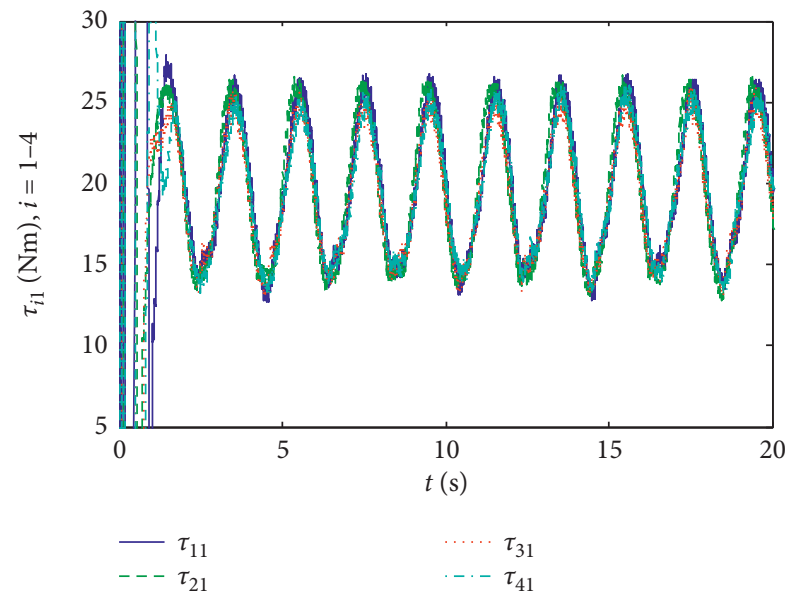

FIgURE 13: The control input torque $\tau_{i 1}, i=1, \ldots, 4$ of manipulators.

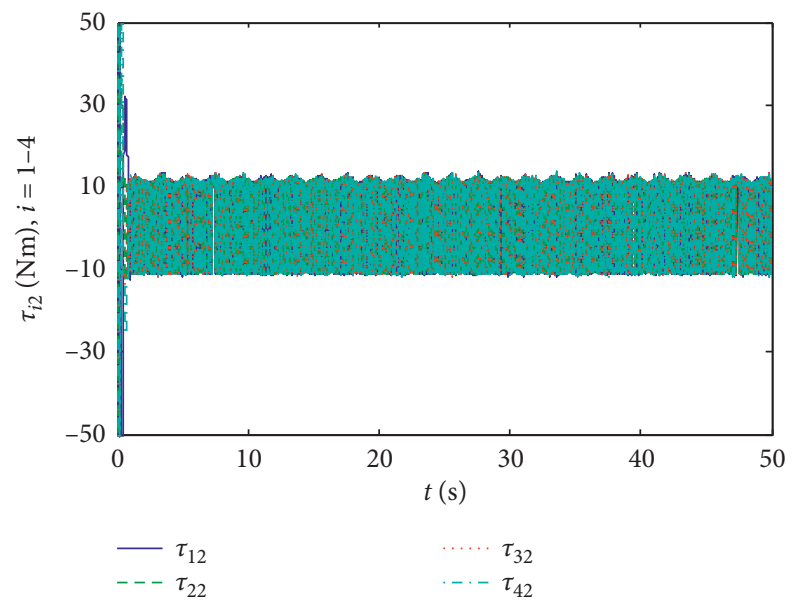

FIgURE 14: The control input torque $\tau_{i 2}, i=1, \ldots, 4$ of manipulators. 


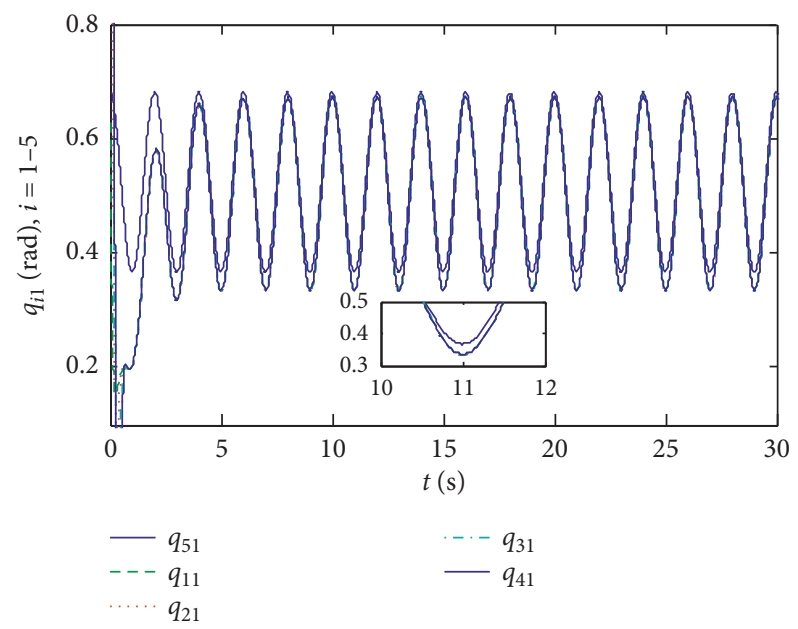

FIgURE 15: The generalized coordinates $q_{i 1}, i=1, \ldots, 4$ of manipulators.

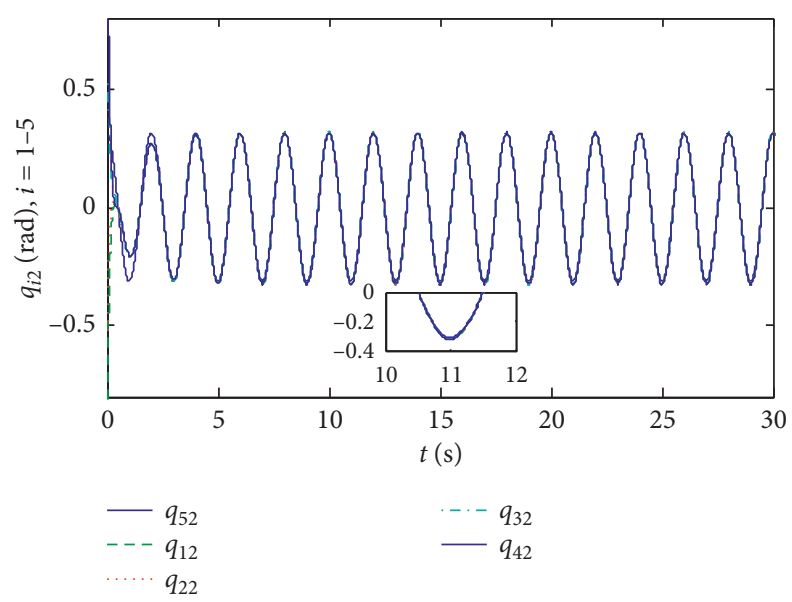

FIgURe 16: The generalized coordinates $q_{i 2}, i=1, \ldots, 4$ of manipulators.

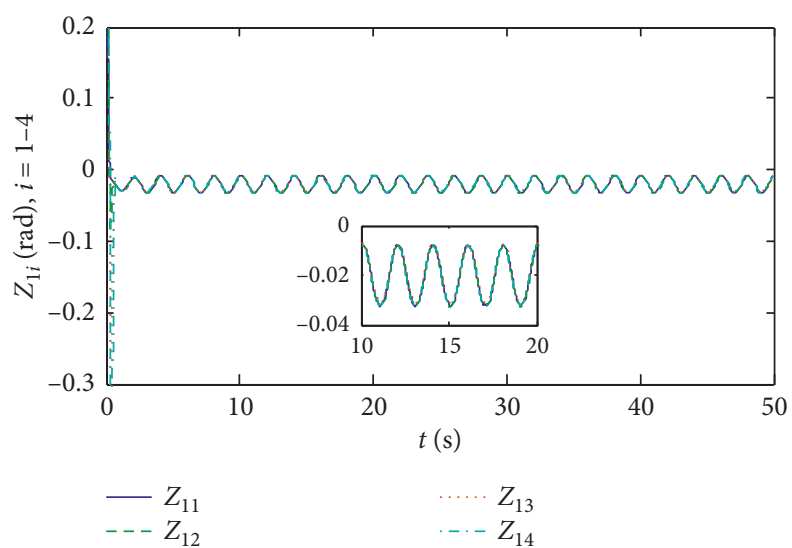

Figure 17: The auxiliary variables $Z_{1 i}, i=1, \ldots, 4$. 


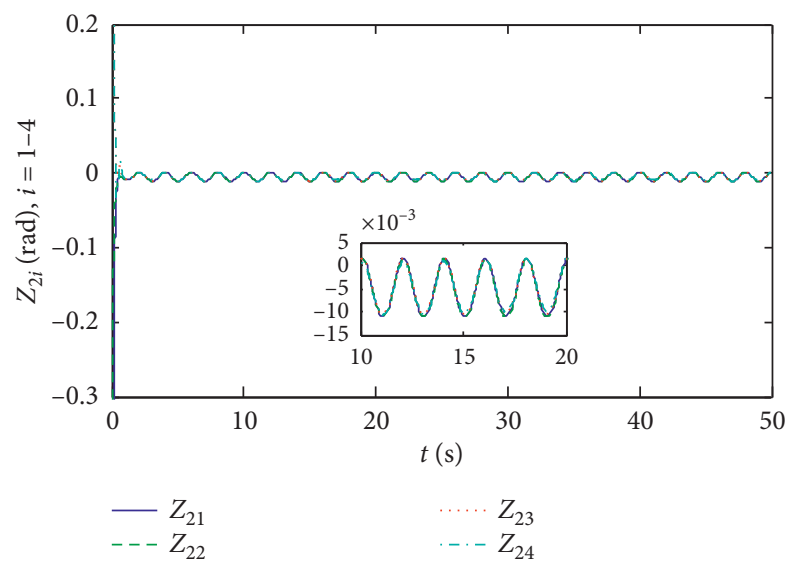

FigURE 18: The auxiliary variables $Z_{2 i}, i=1, \ldots, 4$.

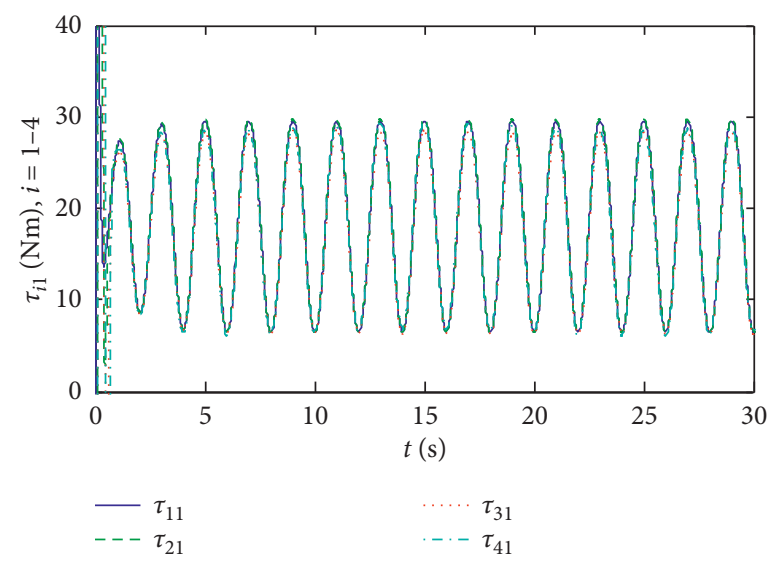

FIgURE 19: The control input torque $\tau_{i 1}, i=1, \ldots, 4$ of manipulators.

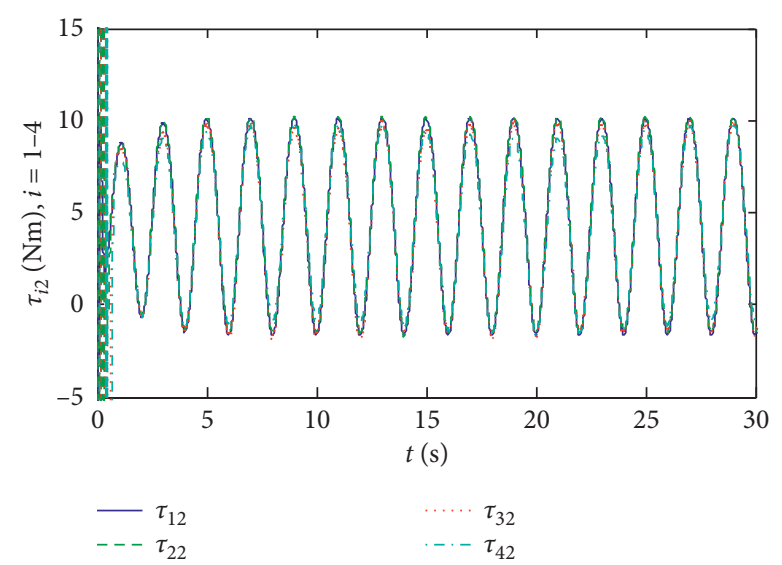

Figure 20: The control input torque $\tau_{i 2}, i=1, \ldots, 4$ of manipulators. 


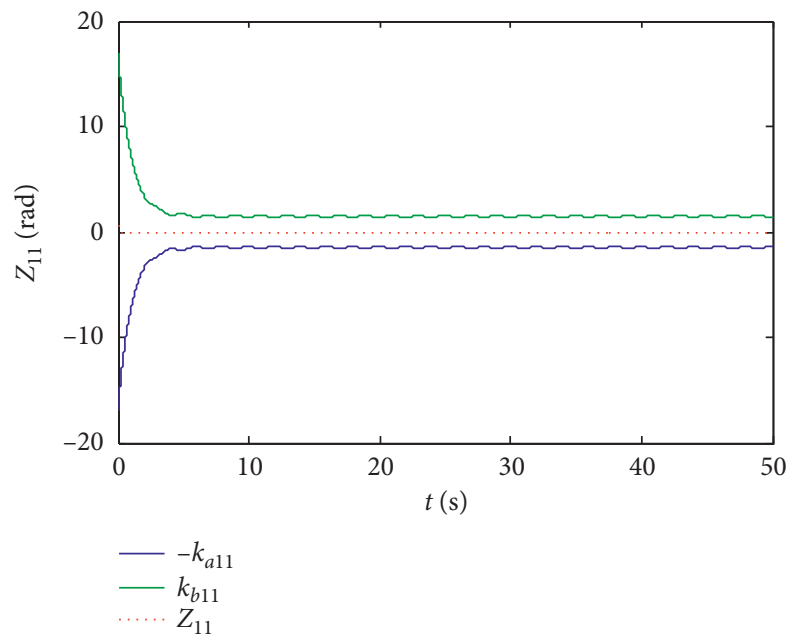

Figure 21: Constraint effect of $Z_{11}$ under Algorithm 2.

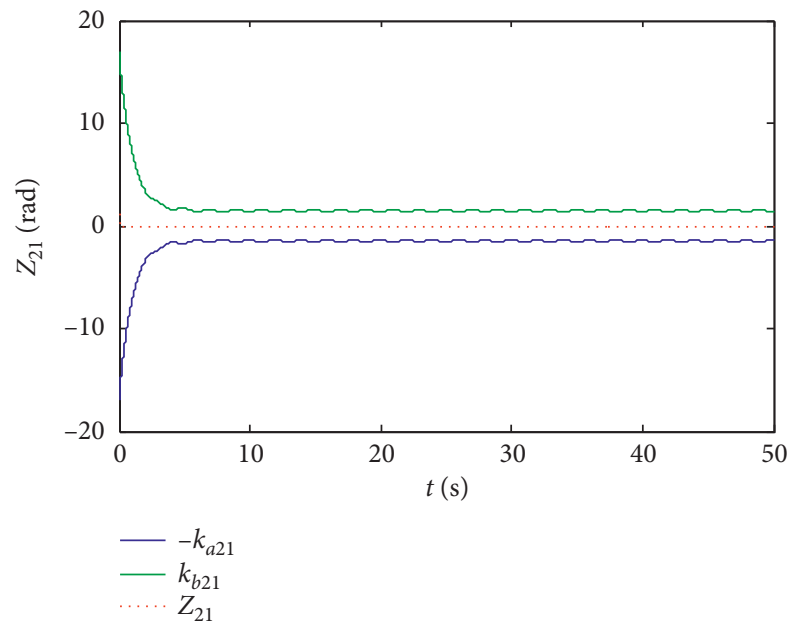

Figure 22: Constraint effect of $Z_{21}$ under Algorithm 2.

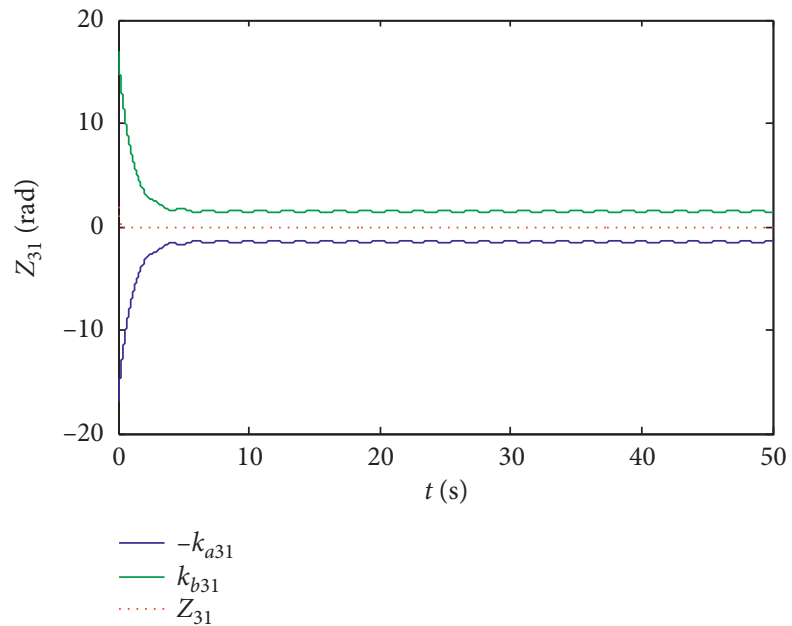

Figure 23: Constraint effect of $Z_{31}$ under Algorithm 2. 


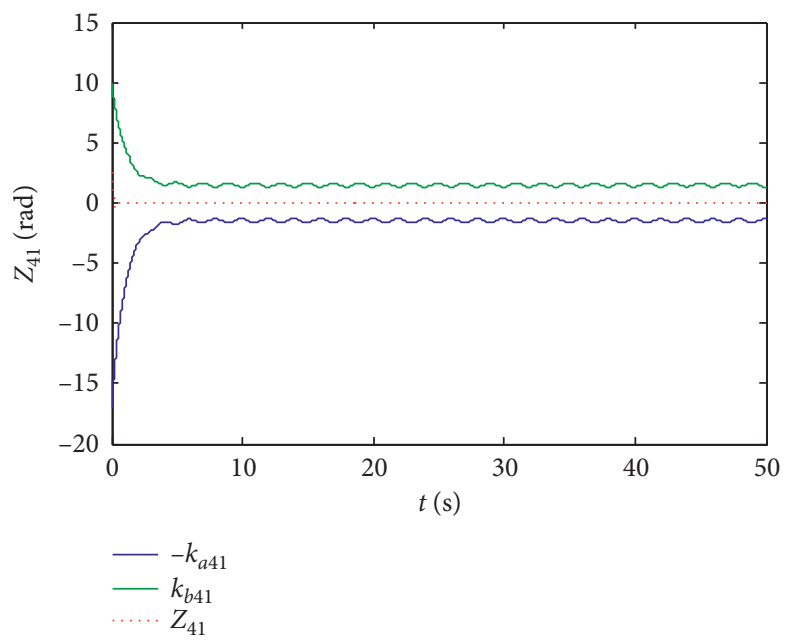

Figure 24: Constraint effect of $Z_{41}$ under Algorithm 2.

and the tracking error always satisfies the time-varying constraints. In addition, the tracking errors $Z_{1 i}$ based on Algorithm 2 are smaller than those based on Algorithm 1, and Algorithm 2 avoids the unexpected chattering problem arising from sign function.

\section{Conclusions}

In this study, we propose two practical control strategies to address distributed coordinated tracking problem for the multiple EL systems subjected to communication delays and time-varying constraints. The distributed observer is used to cope with the communication delays for multiple EL systems. We utilize the NN technique to compensate nonlinear uncertainties. At the same time, an asymmetric BLF is used to guarantee that the output errors are always within the output constraints. The adaptive control Algorithm 1 is designed to ensure that the tracking errors is designed to ensure that the tracking errors among the followers and the virtual leader can be bounded. Based on Algorithm 1, the improved Algorithm 2 can make the tracking errors smaller. The simulation results indicate that the proposed methods can effectively solve the problem of communication delays and make the tracking errors meet the prescribed output constraints.

\section{Data Availability}

The data used to support the findings of this study are included within the article.

\section{Conflicts of Interest}

The authors declare that they have no conflicts of interest.

\section{Acknowledgments}

This study was supported by the National Natural Science Foundation of China (grant nos. 61803119, U1713205, and 51779058).

\section{References}

[1] Y. Jiang, Y. Zhang, and S. Wang, "Distributed adaptive consensus control for networked robotic manipulators with time-varying delays under directed switching topologies," Peer-to-Peer Networking and Applications, vol. 12, no. 6, pp. 1705-1715, 2019.

[2] Y. Xu, D. Luo, D. Li, Y. You, and H. Duan, "Target-enclosing affine formation control of two-layer networked spacecraft with collision avoidance," Chinese Journal of Aeronautics, vol. 32, no. 12, pp. 2679-2693, 2019.

[3] J. Yan, J. Gao, X. Yang, X. Luo, and X. Guan, "Position tracking control of remotely operated underwater vehicles with communication delay," IEEE Transactions on Control Systems Technology, vol. 28, no. 6, pp. 2506-2514, 2020.

[4] L. Shan and C.-L. Liu, "Average-consensus tracking of multiagent systems with additional interconnecting agents," Journal of the Franklin Institute, vol. 355, no. 17, pp. 89578970, 2018.

[5] X. He and Q. Wang, "Distributed finite-time leaderless consensus control for double-integrator multi-agent systems with external disturbances," Applied Mathematics and Computation, vol. 295, pp. 65-76, 2017.

[6] H. Yu and X. Xia, "Adaptive leaderless consensus of agents in jointly connected networks," Neurocomputing, vol. 241, pp. 64-70, 2017.

[7] Q. Wang and C. Sun, "Coordinated tracking of linear multiagent systems with input saturation and stochastic disturbances," ISA Transactions, vol. 71, no. 1, pp. 3-9, 2017.

[8] Y. Xie and Z. Lin, "Global leader-following consensus of a group of discrete-time neutrally stable linear systems by event-triggered bounded controls," Information Sciences, vol. 459, pp. 302-316, 2018.

[9] K. Mouna, M. Mosbah, and A. H. Kacem, "Electing a leader in dynamic networks using mobile agents and local computations," Procedia Computer Science, vol. 109, pp. 351-358, 2017.

[10] M. Guinalíu and P. Jordán, "Building trust in the leader of virtual work teams," Spanish Journal of Marketing-ESIC, vol. 20, no. 1, pp. 58-70, 2016.

[11] Z. Fan, H. Su, S. Chen, M. Yu, and J. Ma, "Semi-global leaderfollowing coordination of multi-agent systems with input saturation and aperiodic intermittent communications," 
Journal of the Franklin Institute, vol. 356, no. 2, pp. 1051-1066, 2019.

[12] T. Chen, H. Wen, H. Hu, and D. Jin, "Distributed finite-time tracking for a team of planar flexible spacecraft," ISA Transactions, vol. 69, pp. 214-221, 2017.

[13] H. Wang, Y. Zou, P. X. Liu, and X. Liu, "Robust fuzzy adaptive funnel control of nonlinear systems with dynamic uncertainties," Neurocomputing, vol. 314, pp. 299-309, 2018.

[14] Q. Yang, M. Cao, H. Garcia de Marina, H. Fang, and J. Chen, "Distributed formation tracking using local coordinate systems," Systems \& Control Letters, vol. 111, pp. 70-78, 2018.

[15] C. Barbălată, M. W. Dunnigan, and Y. Pétillot, "Reduction of the dynamic coupling in an underwater vehicle-manipulator system using an inverse dynamic model approach," IFAC PapersOnLine, vol. 48, no. 2, pp. 44-49, 2015.

[16] Y.-C. Liu, S.-Y. Liu, and N. Wang, "Fully-tuned fuzzy neural network based robust adaptive tracking control of unmanned underwater vehicle with thruster dynamics," Neurocomputing, vol. 196, pp. 1-13, 2016.

[17] H. Wang, "Flocking of networked uncertain Euler-Lagrange systems on directed graphs," Automatica, vol. 49, no. 9, pp. 2774-2779, 2013.

[18] J. Mei, W. Ren, and G. Ma, "Distributed coordinated tracking with a dynamic leader for multiple Euler-Lagrange systems," IEEE Transactions on Automatic Control, vol. 56, no. 6, pp. 1415-1421, 2011.

[19] D. Li, G. Ma, W. He, W. Zhang, C. Li, and S. S. Ge, "Distributed coordinated tracking control of multiple EulerLagrange systems by state and output feedback," IET Control Theory \& Applications, vol. 11, no. 14, pp. 2213-2221, 2017.

[20] P. Li, S. Xu, Q. Ma, W. Chen, and Z. Zhang, "Leader-following rendezvous for uncertain Euler-Lagrange multi-agent systems by output feedback," Journal of the Franklin Institute, vol. 354, no. 10, pp. 4215-4230, 2017.

[21] F. Gao, S. E. Li, Y. Zheng, and D. Kum, "Robust control of heterogeneous vehicular platoon with uncertain dynamics and communication delay," IET Intelligent Transport Systems, vol. 10, no. 7, pp. 503-513, 2016.

[22] X. Ge, Q.-L. Han, and X.-M. Zhang, "Achieving cluster formation of multi-agent systems under aperiodic sampling and communication delays," IEEE Transactions on Industrial Electronics, vol. 65, no. 4, pp. 3417-3426, 2018.

[23] M. B. Lu and L. Lu, "Leader-following consensus of multiple uncertain Euler-Lagrange systems subject to communication delays and switching networks," IEEE Transactions on Automatic Control, vol. 63, no. 8, pp. 2604-2611, 2017.

[24] Y. Liu, S. C. Wang, H. B. Min, and Z. G. Liu, "Distributed adaptive consensus algorithm of Euler-Lagrange system with input and communication delays," Control Theory and Applications, vol. 30, no. 2, pp. 156-162, 2013.

[25] K. Zhao, Y. D. Song, C. L. P. Chen, and L. Chen, "Control of nonlinear systems under dynamic constraints: a unified barrier function-based approach," Automatica, vol. 56, no. 9, pp. 1-9, 2020.

[26] W. Wei and C. Wen, "Adaptive actuator failure compensation control of uncertain nonlinear systems with guaranteed transient performance," Automatica, vol. 46, pp. 2082-2091, 2010.

[27] K. Zhao, Y. Song, T. Ma, and L. He, "Prescribed performance control of uncertain Euler-Lagrange systems subject to fullstate constraints," IEEE Transactions on Neural Networks and Learning Systems, vol. 29, no. 8, pp. 3478-3489, 2018.

[28] W. Zhao, Y. Liu, and L. Liu, "Observer-based adaptive fuzzy tracking control using integral barrier Lyapunov functionals for A nonlinear system with full state constraints," IEEE/CAA Journal of Automatica Sinica, vol. 8, no. 3, pp. 617-627, 2021.

[29] C. Wang, Y. Wu, and J. Yu, "Barrier Lyapunov functionsbased adaptive control for nonlinear pure-feedback systems with time-varying full state constraints," International Journal of Control, Automation and Systems, vol. 15, no. 6, pp. 2714-2722, 2017.

[30] W. He, Z. Yin, and C. Sun, "Adaptive neural network control of a marine vessel with constraints using the asymmetric barrier Lyapunov function," IEEE Transactions on Cybernetics, vol. 47, no. 7, pp. 1641-1651, 2017.

[31] S. Li, L. Ding, and H. B. Gao, "Adaptive neural network tracking control-based reinforcement learning for wheeled mobile robots with skidding and slipping," Neurocomputing, vol. 283, pp. 20-30, 2018.

[32] Q. Yin, M. Wang, X. Li, and G. Sun, "Neural network adaptive tracking control for a class of uncertain switched nonlinear systems," Neurocomputing, vol. 301, pp. 1-10, 2018.

[33] N. B. Hoang and H. J. Kang, "Neural network-based adaptive tracking control of mobile robots in the presence of wheel slip and external disturbance force," Neurocomputing, vol. 188, pp. 12-22, 2016.

[34] Y. Pan, Y. Liu, B. Xu, and H. Yu, "Hybrid feedback feedforward: an efficient design of adaptive neural network control," Neural Networks, vol. 76, pp. 122-134, 2016.

[35] H. Cai and J. Huang, "Leader-following consensus of multiple uncertain Euler-Lagrange systems under switching network topology," International Journal of General Systems, vol. 43, no. 34, pp. 294-304, 2014.

[36] Z. Chen, Z. Li, and C. L. P. Chen, "Adaptive neural control of uncertain MIMO nonlinear systems with state and input constraints," IEEE Transactions on Neural Networks and Learning Systems, vol. 28, no. 6, pp. 1318-1330, 2017.

[37] S. S. Ge, T. H. Lee, and C. J. Harris, Adaptive Neural Network Control of Robotic Manipulators, World Scientific Series in Robotics and Intelligent Systems, London, UK, 1998.

[38] Z. Li, C.-Y. Su, L. Wang, Z. Chen, and T. Chai, "Nonlinear disturbance observer-based control design for a robotic exoskeleton incorporating fuzzy approximation," IEEE Transactions on Industrial Electronics, vol. 62, no. 9, pp. 5763-5775, 2015.

[39] Z. Zhao, W. He, and S. S. Ge, "Adaptive neural network control of a fully actuated marine surface vessel with multiple output constraints," IEEE Transactions on Control Systems Technology, vol. 22, no. 4, pp. 1536-1543, 2014.

[40] W. He, H. Huang, and S. S. Ge, "Adaptive neural network control of a robotic manipulator with time-varying output constraints," IEEE Transactions on Cybernetics, vol. 47, no. 10, pp. 3136-3147, 2017.

[41] Z. Meng, W. Ren, Y. Cao, and Z. You, "Leaderless and leaderfollowing consensus with communication and input delays under a directed network topology," IEEE Transactions on Systems, Man, and Cybernetics, Part B (Cybernetics), vol. 41, no. 1, pp. 75-88, 2011.

[42] Y. Sun, L. Chen, G. Ma, and C. Li, "Adaptive neural network tracking control for multiple uncertain Euler-Lagrange systems with communication delays," Journal of the Franklin Institute, vol. 354, no. 7, pp. 2677-2698, 2017. 